\title{
A Parameter Estimation Technique for a Groundwater Flow Model
}

\author{
Joseph Acquah ${ }^{1,}$, Francis Benyah ${ }^{2}$, Jerry Samuel Yao-Kuma ${ }^{3}$ \\ ${ }^{1}$ Mathematical Sciences Department, University of Mines and Technology (UMaT), Tarkwa, Ghana \\ ${ }^{2}$ Mathematics and Statistics Department, University of Cape Coast (UCC), Cape Coast, Ghana \\ ${ }^{3}$ Geological Engineering Department, University of Mines and Technology (UMaT), Tarkwa, Ghana
}

\section{Email address:}

jacquah@umat.edu.gh (J. Acquah)

${ }^{*}$ Corresponding author

\section{To cite this article:}

Joseph Acquah, Francis Benyah, Jerry Samuel Yao-Kuma. A Parameter Estimation Technique for a Groundwater Flow Model. Mathematical Modelling and Applications. Vol. 5, No. 4, 2020, pp. 202-213. doi: 10.11648/j.mma.20200504.11

Received: August 31, 2020; Accepted: October 12, 2020; Published: December 16, 2020

\begin{abstract}
In this paper, the problem of ill-posedness of solution in identifying multiple groundwater flow parameters from hydraulic head data and other ancillary data was assessed. The solution approach to the parameter identification problem is sought by applying the Least Squares, the Adjoint, the Conjugate Gradient Method and a proposed Parameter Transformation Method. Numerical test for a 1D and 2D flow models governed by PDEs were used to assess the accuracy and stability of the proposed method. The proposed method gave an appreciable solution estimates with minimal error-norm compared with the $o$ ptimisation techniques explored in the study as a measure to the PTM The results revealed that when the adapted methods and the PTM were simulated numerically on a 1D and 2D test problems, the PTM gave a more stable solution estimates with a residual norm-error value of 2.23500 for the $1 \mathrm{D}$ test problem compared with that of the Adjoint method which prove to be the comparing solution with a norm-error value of 2.66500. For the $2 \mathrm{D}$ test case, the results also revealed that the PTM was stable with a residual norm-error value of 10.98310 compared with that of the Conjugate Gradient method with value of 86.562. Thus in conclusion, the study revealed that the PTM is capable of yielding realistic solution estimates compared with the studied optimisation methods.
\end{abstract}

Keywords: Ill-Posed Problem, Parameter Transformation Method, Optimisation Techniques

\section{Introduction}

Parameter Identification refers to the estimation of unknown coefficients in a differential equation describing a phenomenon from observations of the solution to its state variable. The process of assigning appropriate values to model parameters in a given differential equation by comparing model outputs to field measurements is termed as inverse modelling [21]. In groundwater flow, model output refers to hydraulic head, whilst model inputs correspond to drawdown, hydraulic conductivity or transmissivity, recharge, storativity, boundary conditions setting, etc. Most of these output and inputs parameters are determined either by conducting pumping tests or using studied optimisation algorithm to estimate the parameters of interest.

The difficulties associated with the estimation of these flow parameters out of partial differential equations governing a flow model in a bounded region can be attributed to the sparse distribution of hydraulic head data at well sites, as well as inherent measurement errors. Most of the parameters are also very difficult to measure since the bounded regions are inaccessible, resulting in data perturbation. The problem of estimating these parameters are characterised by nonuniqueness of solution due to data insufficiency [1, 6, 27]. A range of algorithms (e.g. PEST, GLUE, DREAM) have been developed to explore the non-uniqueness of model solutions [27]. Alternatively, adjoint state solutions and other gradient search algorithms have also been used.

Though many inverse solution methods for addressing the problem have been proposed, no published works claim to handle fully inverse parameter estimation problem or simultaneous recovery of multiple groundwater flow parameters $[9,14,23]$. Other related studies also bring to 
bear the fact that multiple parameter estimation is possible but, the ill-posedness in the solution turns to increase as the number of parameters to be estimated increases [1].

This paper seeks to develop a novel and efficient method for estimating multiple flow parameters for an inverse groundwater flow model from hydraulic head data. Furthermore, the work would seek to assess the problem of non-uniqueness of solution.

\section{The Inverse Problem Formulation}

The section focuses on the inverse problem formulation for a flow model problem. The exact approach applied to the inverse methodology and the ill-posedness of the inverse problem are discussed under this section.

\subsection{The Model Problem}

Consider the nonlinear partial differential equation for three-dimensional confined groundwater flow model

$$
\begin{aligned}
& \frac{\partial}{\partial x}\left(T(x, y, z) \frac{\partial h}{\partial x}\right)+\frac{\partial}{\partial y}\left(T(x, y, z) \frac{\partial h}{\partial y}\right) \\
& +\frac{\partial}{\partial z}\left(T(x, y, z) \frac{\partial h}{\partial z}\right)=S(x, y, z) \frac{\partial h}{\partial t}+Q
\end{aligned}
$$

Subject to the initial and boundary conditions:

$$
\begin{gathered}
\left.h(x, y, z, t)\right|_{t=0}=h_{0}(x, y, z), \quad(x, y) \in \Omega \\
\left.h(x, y, z, t)\right|_{\Gamma_{1}}=h_{1}(x, y, z, t), \\
\left.T \nabla h(x, y, z, t)\right|_{\Gamma_{2}}=h_{2}(x, y, z, t),
\end{gathered}
$$

where $h=$ Hydraulic head (LT-1), $T=$ transmissivity (LT-1), $S=$ Storage coefficient (unitless), $Q=$ source/sink term (LT1 ), and $t=$ time component. In this problem, $\mathrm{T}$ and $\mathrm{S}$ are the parameters of interest, and $h$ is the state response variable. $\Gamma_{1}$ is the boundary of the aquifer subject to a Dirichlet type boundary conditions and $\Gamma_{2}$ the boundary of the aquifer subject to a Neuman type boundary conditions with flow region $\Omega$. The functions $h_{0}, h_{1}$ and $h_{2}$ are known functions at the boundaries $\Gamma_{1}$ and $\Gamma_{2}$ of the aquifer.

Given the initial and final boundary conditions together with the parameters $\mathrm{T}$ and $\mathrm{S}$ Equations (1) to (4) can be solved numerically to determine the hydraulic head $h(x, y, z, t)$ uniquely. This constitutes the forward problem, but often, the parameters of interest are not known. The inverse problem arises when $h(x, y, z, t)$ is observed at some selected discrete points $\left(x_{i}, y_{i}, z_{i}\right) \in \Omega$ and one is required to solve Equations (1) to (4), to determine one or more of the unknown parameters $\mathrm{T}$ and $\mathrm{S}$. The objective of the inverse problem is to find the parameter $T$ and $S$, so that the head $h$, predicted by the model solution are very close to the observed heads $\bar{h}$, measured by fieldwork. This can be written as an optimisation problem, where the objective is to minimise the squares of the difference between estimated and measured heads as in Equation (5)

$$
\phi=\|\tilde{h}-h\|_{2}^{2}
$$

To estimate the coefficients parameters $\mathrm{T}$ and $\mathrm{S}$, hydraulic heads are measured at discrete points for the parameters $(\mathrm{T}$, S), which is usually not known but determined by conducting pumping tests. The selection criterion is usually based on the minimisation of some function of $(T-\bar{T})$ and $(S-\bar{S})$ using least squares criteria as in Equation (6) over a bounded region $\Omega \subset \mathbb{R}^{n}(n \geq 2)$, with smooth boundary $\partial \Omega$

$$
\phi(T, S)=[\tilde{h}(T, S)-h(\bar{T}, \bar{S})]^{2},
$$

where $h(\bar{T}, \bar{S})$ and $\hat{h}(T, S)$ are the calculated and the observed heads respectively. For an aquifer area $\Omega$ and at time interval $t$, Equation (6) can be expressed mathematically as an integral function as shown by Equation (7)

$$
\phi(T, S)=\int_{t} \int_{\Omega}[\tilde{h}(T, S)-h(\bar{T}, \bar{S})]^{2} d \Omega d t
$$

However, since the hydraulic head are measured at discrete points for $\mathrm{N}$ number of observations over a total time period Tf, the Equation (7) then becomes Equation (8).

$$
\phi(T, S)=\sum_{i=1}^{T_{f}} \sum_{n=1}^{N}\left[\tilde{h}_{t, n}(T, S)-h_{t, n}(\bar{T}, \bar{S})\right]^{2}
$$

The discretisation method leading to Equation (8) almost invariably makes the problem ill-posed. That is, a small perturbation within the data can lead to a large difference in the solution estimates

\subsection{Ill-Posedness of the Inverse Problem}

The associated Inverse Problem stated in Section 2 above is to find $T(x, y)$ when $h(x, y)$ is known or observed from field measurement. To determine analytically the solution to the problem, Equation (1) can be written in linear differential form as stated in Equation (9)

$$
a \frac{\partial T}{\partial x}+b \frac{\partial T}{\partial y}+c T-Q=0
$$

where $a=\frac{\partial h}{\partial x}, b=\frac{\partial h}{\partial y}$, and $c=\frac{\partial^{2} h}{\partial x^{2}}+\frac{\partial^{2} h}{\partial y^{2}}$. The variables $a, b, c$ and $h(x, y)$ are known functions. Equation (9) is a first order PDE with respect to Transmissivity $T(x, y)$. The general solution of Equation (9) involve arbitrary functions of $\mathrm{T}$ and has the characteristic equation given by Equation (10) 


$$
\frac{d x}{a}=\frac{d y}{b}=\frac{d T}{(Q-c T)}
$$

Equation (10) has at least two or more solutions. If $T_{1}(x, y)$ and $T_{2}(x, y)$ are solutions generated out of Equation (10), then $T_{1}+T_{2}$ is also a solution of the Equation (10). Therefore, the solution of the inverse problem in Equation (1) is not unique. To assess the stability of the problem, the flow Equation in (1) and its boundary conditions can be subjected to the Cauchy data in Equation (11)

$$
\left.T \frac{\partial h}{\partial x}\right|_{x}=-q_{1}, \quad \text { and }\left.\quad T \frac{\partial h}{\partial y}\right|_{y}=-q_{2} ; q>0
$$

From Equation (1), the forward problem of finding $h(x, y)$ given $T(x, y)$ can be determined by Direct Integration of Equation (1) using the Cauchy data in Equation (11). Assuming that $T^{*}(x, y)=T(x, y)+\varepsilon(x, y)$, where $\varepsilon(x, y)$ is an error term in the known value of $T$, then from Equation (1), one obtains Equations (12) and (13)

$$
\begin{array}{r}
\left|h_{x}-h_{x}^{*}\right|=q_{1} \int_{x_{1}}^{x} \frac{\varepsilon(x, y) d x}{T(x, y)[T(x, y)+\varepsilon(x, y)]} \\
\left|h_{y}-h_{y}^{*}\right|=q_{2} \int_{y_{1}}^{y} \frac{\varepsilon(x, y) d y}{T(x, y)[T(x, y)+\varepsilon(x, y)]}
\end{array}
$$

Combining Equations (12) and (13), gives Equation (14)

$$
\left|h(x, y)-h^{*}(x, y)\right|=M|\mathcal{E}(x, y)|+N|\mathcal{E}(x, y)|
$$

where $\mathrm{M}$ and $\mathrm{N}$ are arbitrary constants and defined respectively as

$$
M(x, y)=q_{1} \int_{x_{1}}^{x} \frac{1}{T(x, y)[T(x, y)+\varepsilon(x, y)]} d x
$$

and

$$
N(x, y)=q_{2} \int_{y_{1}}^{y} \frac{1}{T(x, y)[T(x, y)+\varepsilon(x, y)]} d y .
$$

Thus, substituting the arbitrary constants $\mathrm{M}$ and $\mathrm{N}$ into Equation (14) yields:

$$
\left|h(x, y)-h^{*}(x, y)\right| \leq K(x, y)|\varepsilon(x, y)|
$$

where $K(x, y)=M(x, y)+N(x, y)$. The Equation (15) signifies that the forward problem is very stable. But in this identification problem, one's intent is to solve the inverse problem for $T(x, y)$ given $h(x, y)$. Because the Cauchy data is already given by the boundary conditions, the inverse problem has unique solution given as in Equation (16)

$$
\left.\begin{array}{l}
T_{x}(x, y)=-\frac{q_{1}}{\partial h / \partial x} \\
T_{y}(x, y)=-\frac{q_{2}}{\partial h / \partial y}
\end{array}\right\}
$$

The true distribution of $h(x, y)$ (or the exact value of the head distributions) can never be known. Thus, one can only have noisy head observations: $h^{*}(x, y)=h(x, y)+\eta(x, y)$, where $\eta(x, y)$ is the error term in the head data. The corresponding inverse solution from Equation (16) now becomes Equation (17)

$$
\left.\begin{array}{l}
T_{x}^{*}(x, y)=-q_{1} /\left(\frac{\partial h}{\partial x}+\frac{\partial \eta}{\partial x}\right) \\
T_{y}^{*}(x, y)=-q_{2} /\left(\frac{\partial h}{\partial y}+\frac{\partial \eta}{\partial y}\right)
\end{array}\right\}
$$

Subtracting Equations (17) from (16), by taking absolute of both sides, gives Equation (18)

$$
\begin{aligned}
\left|T(x, y)-T^{*}(x, y)\right| & =\frac{q_{1}|\partial \eta / \partial x|}{|\partial h / \partial x(\partial h / \partial x+\partial \eta / \partial x)|} \\
& +\frac{q_{2}|\partial \eta / \partial y|}{|\partial h / \partial y(\partial h / \partial y+\partial \eta / \partial y)|}
\end{aligned}
$$

From Equation (18), it can be inferred that although $|\eta(x, y)|$ is very small, $|\partial \eta / \partial x|$ and $|\partial \eta / \partial y|$ are very large. Thus, from Equation (18), the solutions to Equation (1) is generally not unique and unstable to the observation error. To conclude, the non-steady state 2-D groundwater flow equation is ill-posed in nature.

\section{The Solution Methods}

The solution methods considered for this study are: The Least Squares Method [16], the Adjoint Method [25, 26], and the proposed Parameter Transformation Method (PTM). A Finite Difference Scheme by [11] was used to discretise the flow equation in Equation (1) to obtain the parameter dependent system in Equation (19).

$$
A(q) h=F ; \quad \text { with } q=[S, T]
$$

where $A(q)$ is an operator matrix that depends on the vector of unknown $q, \mathrm{~h}$ is observed hydraulic head data, and $\mathrm{F}$ is a vector of functions that are not hydraulic head dependent and encompass also the boundary conditions. A detailed approach of how the parameter dependent system in Equation (19) was obtain is demonstrated in the text by two numerical examples. The matrix $A(q)$ is a symmetric positive definite and a block tridiagonal matrix. 
The inverse problem of interest is to estimate $q$ in Equation (19) given the observed data $h$ from field measurements. For this study, the following solution methods were considered to estimate the parameter of interest.

\subsection{Solution by Least Squares Method}

The main idea here is to minimise the error function $\mathrm{E}$ as a function of the parameter $q$ such that a measure of the difference between the estimated $\left(h_{q}\right)$ and observed data $\left(h_{o b s}\right)$ is a minimum [16]. A necessary condition for the minimum value of $E(q)$ is that the derivative of $\mathrm{E}$ with respect to $q$ becomes zero.

$$
\frac{\partial \phi}{\partial q}=2 \sum\left[h_{q}(x, y, \mathrm{t})-h_{o b s}(x, y, t)\right] \frac{\partial h}{\partial q}=0
$$

The computational procedures for recovering the parameter is by "try and error" method. First, an initial guess of $q_{i}$ is chosen based on the stability criteria of the finite difference scheme. An error term $\phi_{i}$ expressed as in Equation (21).

$$
\phi_{i}=h_{q}(x, y, t)-h_{o b s}(\mathrm{x}, \mathrm{y}, \mathrm{t}), \forall i=1,2,3, \ldots, n
$$

is determined such that the difference between the observed and the estimated minimises the function as shown in Equation (22)

$$
\phi\left(q_{1}, q_{2}, q_{3}, \ldots, q_{n}\right)=\sum_{j=1}^{n}\left(\phi_{j}\right)^{2}
$$

To modify $q_{i}$ to increase its convergence, a new value $h_{q}^{*}(x, y, t)$ is sought based on the initial observed value $h_{q}$. The new value is obtained by approximating the function $u$ with the derivative function which determines an increase or decrease as indicated in Equation (23)

$$
h_{q}^{*}=h_{q}+\sum_{i=0}^{n}\left(\frac{\partial h_{q}}{\partial q_{i}^{*}}\right) \cdot u_{i}
$$

where $u_{i}$ represent the change between successive intervals for the value of $q_{i}$. Then the error $\phi_{j}^{*}$ in the new estimated value $h_{q}^{*}$ can also be expressed as:

$$
\phi_{j}^{*}=h_{q}^{*}-h_{o b s} \forall j=1,2,3, \ldots, n
$$

The derivative function in Equation (23) can be obtained by difference approximation $(\delta)$. given by Equation (25).

$$
\delta_{j}^{i}=\frac{\partial h_{q}}{\partial q_{i}}=\frac{h_{q}^{*}\left(q_{1}+u_{1}, q_{2}+u_{2}, \ldots ., q_{n}+u_{n}\right)-h_{q}\left(q_{1}, q_{2}, \ldots, q_{n}\right)}{u_{i}}
$$

Substituting Equation (25) into Equation (23) yields Equation (26).

$$
h_{q}^{*}=h_{q}+\sum_{i=0}^{n}\left(\delta_{j}^{i}\right) \cdot u_{i}
$$

From Equations (21), (24) and (26), one obtains Equation (27).

$$
\phi_{j}^{*}=\phi_{j}+\sum_{i=0}^{n}\left(\delta_{j}^{i}\right) \cdot u_{i}
$$

But from Equation (27), the error in the new estimate can also be optimised as in Equation (28).

$$
\phi^{*}\left(q_{1}, q_{2}, \ldots, q_{n}\right)=\sum_{j=1}^{n}\left(\phi_{j}^{*}\right)^{2}=\sum_{j=i}^{n}\left[\phi_{j}+\sum_{i=0}^{n}\left(\delta_{j}^{i}\right) \cdot u_{i}\right]^{2}
$$

To minimise the value of $\phi^{*}$ with respect to $q_{i}$ to recover the exact value of $q$, the expected value $\phi^{*}$ is differentiated with respect to the small change $u_{i}$. The derivative function becomes

$$
\frac{\delta \phi^{*}}{\delta u_{i}}=2 \sum_{j=i}^{n}\left[\phi_{j}+\sum_{i=0}^{n}\left(\delta_{j}^{i}\right) \cdot u_{i}\right] \cdot \sum_{i=0}^{n}\left(\delta_{j}^{q}\right)=0
$$

The derived model corresponding to the value of $q_{i}$ is also given by Equation (30).

$$
\Rightarrow \sum_{j=i}^{n}\left[\sum_{i=0}^{n}\left(\delta_{j}^{q} \cdot \delta_{j}^{i}\right) \cdot u_{i}\right]=-\sum_{i=0}^{n}\left(\phi_{j} \delta_{j}^{q}\right), \forall i=0,1,2, \ldots, n .
$$

The Equation (30) signifies that parameter $\delta_{j}^{q}$ within the model equation (Equation (30)) minimises the error factor to produce an approximate solution for $q$. Usually, computing the gradient of the least squares cost function is extremely difficult. This is because the matrix in question is not a constant matrix. A way out is the Adjoint or Costate Method proposed by [3].

\subsection{Solution by Adjoint Method}

The method was originally formalised by [3] following previous applications in reservoir characterisation $[4,5,8]$; and nuclear engineering [15]. The method was introduced to the groundwater modelling literature by [20], following earlier implementations by $[12,13,24]$. Subsequent publications included [18, 22, 27]. In summary, since the publication of [20], over 40 applications of adjoint methods to confined groundwater flow problems have been published. Formulating the minimisation problem in Equation (1) using adjoint methods requires the computations of the gradient of the least squares function defined in Equation (21).

$$
\phi_{l s}(q)=\left\|h(q)-h_{o b s}\right\|_{2}^{2}=\left\|F(q)-h_{o b s}\right\|_{2}^{2}
$$

where $\quad h_{\text {obs }} \stackrel{\text { def }}{=} C h+\mathfrak{y}=d, \quad \mathfrak{y} \quad$ is noise, $h(q)=F(q)=C A(q)^{-1} f, C$ is the state to observation map 
and $r(q)=F(q)-d$; the residual. The objective is to solve numerically for an optimal parameter using the gradient algorithm

$$
q_{k+1}=q_{k}+\alpha_{k} \nabla \phi_{l s}\left(q_{k}\right)
$$

The component of the gradient $\nabla \phi_{l s}(q)$ of the least squares function $\phi_{l s}(q)$ be expressed as shown in Equation (33).

$$
\left(\nabla \phi_{l s}(q)\right)_{i}=\left\langle\left(\frac{d A}{d q} e_{i}\right) \cdot A(q)^{-1} f, A^{T}(q)^{-1} C^{T} r(q)\right\rangle
$$

If the solution to the state Equation (19) is $u$ and that of the Costate or Adjoint Equation: $A^{T}(q) \mathrm{z}=-C^{T} r(q)$ is $z$, then Equation (34) becomes

$$
\left(\nabla \phi_{l s}(q)\right)_{q}=-\left(C A(q)^{-1} \cdot\left(\frac{d A}{d q} e_{i}\right) \cdot h(q)\right)^{T} r(q)
$$

The matrix operator $A(q)$, has a diagonalisation of the form: $A(q)=B_{\text {div }} D(q) B_{\text {grad }}$, where $B_{\text {div }}=B_{\text {grad }}^{T}$ is the block upper diagonal, and $D(q)$ is the diagonal entries of $q$. Substituting the derivative of $\mathrm{A}$ with respect to $q_{i}$ into Equation (34) and simplifying gives Equation (35).

$$
\left(\nabla \phi_{l s}(q)\right)_{i}=\frac{1}{u}\left\langle\left(\frac{d D}{d q_{i}} e_{i}\right) B^{T} h, B z\right\rangle
$$

The vectors $h$ and $z$ in Equation (36) are the solutions to the discrete state and Costate equations $A(q) h=F$ and $A^{T}(q) \mathrm{z}=-C^{T} r(q)$ respectively.

\subsection{Solution by the Proposed Parameter Transformation Method}

The solution methods for the parameter distributed system discussed in Sections 4 and 5 can sometimes be very intractable and unwieldy. As an alternative, the Parameter Transformation Method (PTM) is proposed. Consider the parameter-dependent linear system problem given in Equation (19).

Given the entries $\left[q_{i j}\right] ; 1<i<m$;

$1<j<n$ of $\mathrm{A}$, and the right-hand vector $F^{T}=\left[\begin{array}{lllll}f_{1} & f_{2} & f_{3} & \ldots & f_{m}\end{array}\right] \quad$, then determining

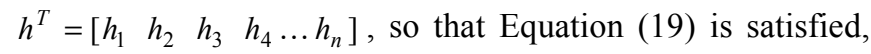
is a forward problem. Suppose the problem posed in Equation (19) is to determine the entries $\left[q_{i j}\right]$ of the matrix operator $A(q)$; given the hydraulic head $h$ and the right-hand vector $F$; then this constitute an inverse problem.

Our approach to solving this inverse problem is based on the Proposition stated below. The proposition transforms Equation (19) into an equivalent linear system, whose solution gives the unknown entries of the matrix $A$ as a vector. The unknown vector can then be solved by standard numerical algorithms.

Proposition: The linear system problem in Equation (19) is equivalent to the linear system in Equation (37).

$$
B(h) q=F,
$$

where $q$ is an $m n \times 1$ vector and $\mathrm{B}$ an $m \times m n$ matrix given by

$$
q=\left[\begin{array}{c}
q_{1 n} \\
q_{2 n} \\
\vdots \\
\vdots \\
q_{m n}
\end{array}\right] \text {, and } B=\left[\begin{array}{ccccc}
h^{T} & 0^{T} & \cdots & \cdots & 0^{T} \\
0^{T} & h^{T} & 0 & \cdots & 0 \\
\vdots & 0^{T} & h^{T} & \ddots & \vdots \\
\vdots & \vdots & \ddots & \ddots & 0^{T} \\
0 & \cdots & \cdots & 0^{T} & h^{T}
\end{array}\right]
$$

The parameters $q, u$ and 0 are defined respectively as:

$$
\begin{gathered}
q_{i n}^{T}=\left[q_{i 1}, q_{i 2}, q_{i 3}, \ldots, q_{i n}\right], h^{T}=\left[h_{1}, h_{2}, h_{3}, \cdots, h_{n}\right] \\
\text { and } 0^{T}=\left[\begin{array}{llll}
0 & 0 & 0 & \cdots
\end{array}\right] \forall i=1,2, \ldots, m
\end{gathered}
$$

Proof

It can be verified from the Proposition that

$$
A(q) h=B(h) q=F
$$

Discretising Equation (1) by Cell Centered Finite Difference (CCFD) scheme gives

$$
\begin{aligned}
& \frac{1}{2}\left(T_{i-1, j}+T_{i, j}\right)\left(h_{i-1, j}^{k}-h_{i, j}^{k}\right)+\frac{1}{2}\left(T_{i+1, j}+T_{i, j}\right)\left(h_{i+1, j}^{k}-h_{i, j}^{k}\right) \\
& +\frac{1}{2}\left(T_{i, j-1}+T_{i, j}\right)\left(h_{i, j-1}^{k}-h_{i, j}^{k}\right)+\frac{1}{2}\left(T_{i, j+1}+T_{i, j}\right)\left(h_{i, j+1}^{k}-h_{i, j}^{k}\right) \\
& =S_{i, j}\left(\frac{h_{i, j}^{k}-h_{i, j}^{k-1}}{\Delta t}\right)(\Delta x)(\Delta y)+Q_{i . j}(\Delta x)(\Delta y)
\end{aligned}
$$

Expressing Equation (38) as a parameter dependent linear system yields

$$
\begin{gathered}
\sum_{i=1}^{n} \sum_{j=1}^{m} L_{i j}[S, T] h_{j}=F \\
\sum_{i=1}^{n} \sum_{j=1}^{m} L_{i j}[q] h_{j}=F
\end{gathered}
$$

where $q=(S, T)$, and $F$ is a function of a space state $\eta$. In compact form Equation (39) becomes:

$$
A(q) h=F
$$

The derivative operator $L_{i j}[q]$ with basis functions $w_{i}, w_{j}$ can be defined as 


$$
\begin{aligned}
\mathrm{L}_{i j}[q] & \stackrel{\operatorname{def}}{=} \sum_{\Omega} q \int_{\Omega} \nabla w_{j} \cdot \nabla w_{i} d \Omega \\
& =\sum_{\Omega} q\left[\operatorname{meas}(\Omega) \nabla w_{j} \cdot \nabla w_{i}\right]=\sum_{\Omega} q^{\Omega} L_{i j}^{\Omega}
\end{aligned}
$$

The Equation (41) is a measure of the parameter unto the domain of the function. The inverse problem of identifying the parameter $q$ can be reconstructed from Equation (42) as

$$
\sum_{1 \leq j \leq m} M_{i j}[h] q_{j}=F ; 1 \leq i \leq n
$$

where $M_{i j}[h] \stackrel{\text { def }}{=} \sum_{\Omega} \int_{\Omega}\left(\nabla h \cdot \nabla w_{j}\right)\left(\nabla h \cdot \nabla w_{i}\right) d \Omega$

The transformed matrix $M_{i j}$ can be recast from $L_{i j}$ using Equation (41) as

$$
M_{i j}[h]=\sum_{\Omega}\left(\sum_{1 \leq k \leq n} h_{k} L_{k i}^{\Omega}\right)\left(\sum_{1 \leq l \leq n} h_{l} L_{l j}^{\Omega}\right)\left(q^{\Omega}\right)^{-1}=B[h]
$$

The equivalent transformation of Equation (40) then becomes $B(h) q=F$.

Suppose we consider the parameter-dependent linear system $A(q) h=f$ for the special case with dimensions $m=5$ and $n=3$, then to transform
Equation (1) into $B(h) q=F$, the parameters in the system can be defined as follows:

$$
\begin{aligned}
& h^{T}=\left[h_{1}, h_{2}, h_{3}\right] \text {, } \\
& q^{T}=\left[\begin{array}{lllll}
\tilde{q}_{13}, & \tilde{q}_{23}, & \tilde{q}_{33}, & \tilde{q}_{43}, & \tilde{q}_{53}
\end{array}\right], \\
& 0^{T}=\left[\begin{array}{lll}
0 & 0 & 0
\end{array}\right] \text { and } \\
& \tilde{q}_{i 3}^{T}=\left[q_{i 1}, \quad q_{i 2}, q_{i 3}\right], i=1,2, \ldots, 5 .
\end{aligned}
$$

The equivalent transformation $B(h) q=F$ is given by Equation (43).

$$
\left[\begin{array}{lllll}
h^{T} & 0^{T} & 0^{T} & 0^{T} & 0^{T} \\
0^{T} & h^{T} & 0^{T} & 0^{T} & 0^{T} \\
0^{T} & 0^{T} & h^{T} & 0^{T} & 0^{T} \\
0^{T} & 0^{T} & 0^{T} & h^{T} & 0^{T} \\
0^{T} & 0^{T} & 0^{T} & 0^{T} & h^{T}
\end{array}\right]\left[\begin{array}{c}
\tilde{q}_{13} \\
\tilde{q}_{23} \\
\tilde{q}_{33} \\
\tilde{q}_{43} \\
\tilde{q}_{53}
\end{array}\right]=\left[\begin{array}{c}
f_{1} \\
f_{2} \\
f_{3} \\
f_{4} \\
f_{5}
\end{array}\right]
$$

Equation (43) can further be expanded as

$$
\left[\begin{array}{ccccccccccccccc}
h_{1} & h_{2} & h_{3} & 0 & 0 & 0 & 0 & 0 & 0 & 0 & 0 & 0 & 0 & 0 & 0 \\
0 & 0 & 0 & h_{1} & h_{2} & h_{3} & 0 & 0 & 0 & 0 & 0 & 0 & 0 & 0 & 0 \\
0 & 0 & 0 & 0 & 0 & 0 & h_{1} & h_{2} & h_{3} & 0 & 0 & 0 & 0 & 0 & 0 \\
0 & 0 & 0 & 0 & 0 & 0 & 0 & 0 & 0 & h_{1} & h_{2} & h_{3} & 0 & 0 & 0 \\
0 & 0 & 0 & 0 & 0 & 0 & 0 & 0 & 0 & 0 & 0 & 0 & h_{1} & h_{2} & h_{3}
\end{array}\right]\left[\begin{array}{c}
q_{11} \\
q_{12} \\
q_{13} \\
q_{21} \\
q_{22} \\
q_{23} \\
q_{31} \\
q_{32} \\
q_{33} \\
q_{41} \\
q_{42} \\
q_{43} \\
q_{51} \\
q_{52} \\
q_{53}
\end{array}\right]=\left[\begin{array}{c}
f_{1} \\
f_{2} \\
f_{3} \\
f_{4} \\
f_{5}
\end{array}\right]
$$

The linear system problem in Equation (44) is underdetermined since the number of equations $m$ is less than the number of unknowns $m n$. However, in most parameter estimation problems the matrix $\mathrm{B}(\mathrm{h})$ is sparse, and as such the number of parameters $q_{i j}$; to be estimated is much less than $m n$. In fact, very often, the number of non-zero entries of $\mathrm{B}(\mathrm{h})$ is less than $m$; resulting in an over-determined linear system, which can be solved by the least-squares method. The Proposition 1 can be used to determine both isotropic and anisotropic conditions

\section{Numerical Test 1}

Consider the problem of identifying the diffusitivity $q(x)$ 
of sediments from measurements of a piezometric head $h$ in a one-dimensional steady-state diffusion equation given by Equation (45). Although, the Numerical Test 1 considered is a well-posed problem, random noise (y) was added to $h$ to make the problem an ill-posed one.

$$
-\frac{\partial}{\partial x}\left(q(x) \frac{\partial h}{\partial x}\right)=f(x), x \in(0,1)
$$

The essence of the numerical example is to ascertain the efficiency of the proposed PTM method. The interval chosen for the model example is $[0,1]$, with homogeneous Dirichlet boundary conditions as stated in Equation (46).

$$
h(0)=0 \text {, and } h(1)=1,
$$

The source terms $f$ for the model problem were taken as Dirac delta $(\delta)$ functions defined as in Equation (47).

$$
\begin{aligned}
& f_{1}(x)=\delta(x-1 / 4), \\
& f_{2}(x)=\delta(x-1 / 2), \\
& f_{3}(x)=\delta(x-3 / 4)
\end{aligned}
$$

The inverse problem of interest is to determine the unknown diffusion coefficient $\mathrm{q}(\mathrm{x})$, given a measurement of the solution $\mathrm{h}(\mathrm{x})$, called the observed data $\left(h_{\text {obs }}\right)$, and the source or $\operatorname{sink} \operatorname{term} f$. The solution approach is to apply the proposed PTM coupled with least squares, and the Adjoint Method. The Adjoint Method was used as a measure to the proposed Parameter Transformation Method (PTM).

Applying standard piecewise linear Finite Difference Discretisation Scheme with nodes $x_{i}=i \Delta x, \Delta x=1 /(n+1), n=50$, to discretise Equation (45), one obtains Equation (48)

$$
-q_{i+1} h_{i+1}+\left(q_{i+1}+q_{i}\right) h_{i}-q_{i} h_{i-1}=(\Delta x)^{2} f_{i}, \quad \forall 1 \leq i \leq n
$$

In compact form Equation (48) simplifies to Equation (19). The operator $A(q)$ generated out of Equation (42) is a diagonalisation of the form

$A(q)=\frac{1}{(\Delta x)^{2}} B^{T} D(q) B$, with $\mathrm{B}$ being an upper block bidiagonal system and $D(q)$ the diagonal system with diagonal entries consisting of $q_{i}$. The parameter dependent operator system $A(q)$ in matrix form is given by Equation (49). Transforming the parameter dependent operator in Equation (19) to a vector dependent operator using the PTM yields Equation (37). The vector dependent operator $B(h)$ in Equation (37) can be formulated out of Equation (45) as shown in Equation (50)

$$
\begin{aligned}
& A(q) h=\left[\begin{array}{ccccccc}
\left(q_{1}+q_{2}\right) & -q_{2} & 0 & 0 & 0 & \ldots & 0 \\
-q_{2} & \left(q_{2}+q_{3}\right) & -q_{3} & 0 & 0 & \ldots & 0 \\
0 & -q_{3} & \left(q_{3}+q_{4}\right) & -q_{4} & 0 & \ldots & 0 \\
0 & 0 & -q_{4} & \left(q_{4}+q_{5}\right) & -q_{5} & \ldots & 0 \\
\vdots & \vdots & \vdots & \ddots & \ddots & \ddots & \vdots \\
0 & 0 & \ldots & 0 & -q_{49} & \left(q_{49}+q_{50}\right) & -q_{50} \\
0 & 0 & \ldots & 0 & 0 & -q_{50} & q_{50}
\end{array}\right]\left[\begin{array}{c}
h_{1} \\
h_{2} \\
h_{3} \\
h_{4} \\
\vdots \\
h_{50} \\
h_{51}
\end{array}\right] \\
& B(h) q=\left[\begin{array}{ccccccc}
h_{1} & \left(h_{1}-h_{2}\right) & 0 & 0 & 0 & \ldots & 0 \\
0 & \left(h_{2}-h_{1}\right) & \left(h_{2}-h_{3}\right) & 0 & 0 & \ldots & 0 \\
0 & 0 & \left(h_{3}-h_{2}\right) & \left(h_{3}-h_{4}\right) & 0 & \ldots & 0 \\
0 & 0 & 0 & \left(h_{4}-h_{3}\right) & \left(h_{4}-h_{5}\right) & \ldots & 0 \\
\vdots & \vdots & \ddots & \ddots & \ddots & \ddots & \vdots \\
0 & 0 & 0 & 0 & \left(h_{49}-h_{48}\right) & \left(h_{49}-h_{50}\right) & 0 \\
0 & 0 & 0 & 0 & 0 & \left(h_{50}-h_{49}\right) & \left(h_{50}-h_{51}\right)
\end{array}\right]\left[\begin{array}{c}
q_{1} \\
q_{2} \\
q_{3} \\
q_{4} \\
\vdots \\
q_{50} \\
q_{51}
\end{array}\right] \\
& \left.\begin{array}{l}
q_{1 \text { true }}=1+\exp \left(-\left(x_{1}-0.25\right)^{2} / 0.01\right) \\
q_{2 \text { true }}=1+\exp \left(-\left(x_{2}-0.5\right)^{2} / 0.01\right) \\
q_{3 \text { true }}=1+\exp \left(-\left(x_{3}-0.75\right)^{2} / 0.01\right)
\end{array}\right\}
\end{aligned}
$$

To generate the head data $(h)$, the forward problem in Equation (19) is solved numerically for the solution $h$ using the parameter values defined in Equation (51). An observed parameter $q$ can be determined form Equation (37) by making use of Equation (45). Usually, the observed data $h_{\text {obs }}$ taken from field measurements are contaminated with noise or are inexact. Suppose an observed data $h_{o b s}$ to the system problem is sought from knowledge of $h$, then the exact parameter value (s) must be defined to enable one estimate $h$. If the parameter value is defined as a function of the source term deviation at different point sources, then the parameter values can be formulated as stated in Equation (51) 
data $h_{\text {obs }}$ to the system is sought by adding random noise (y) to the head data $h$, as indicated in Equation (52).

$$
h_{o b s}=h+\eta
$$

The motive here is to simulate the Modelled parameters given the observed head data $\left(h_{o b s}\right)$. Approximate solution depends on minimum norm solution by the method of least squares. The least squares minimisation function for the model equation is given by Equation (5). The observed head data $\left(h_{o b s}\right)$ and the true head data $(h)$, at discrete and different point sources for the one-dimensional steady-state problem are shown in Figure 1. From Figure 1, the left, the middle and the right curves represent the solution corresponding to the point sources $x_{1}=\frac{1}{4}, x_{2}=\frac{2}{4}$ and $x_{3}=$ $\frac{3}{4}$ respectively. The true solution for each point source are represented by a dashed line, whilst the observed is by a circle. The observed and the true data modelled do not gives a perfect curve fit. Using the generated observed data and the forcing functions given in Equation (51), at a point source of $x_{2}=1 / 2$, the unknown parameter $q$ for the $1 \mathrm{D}$ steady-state diffusion equation can be determined by both methods; either PTM or and the Costate Method. For the PTM, the transformed equation in Equation (37) was used to simulate for the unknown parameter of interest. The Adjoint method was also used as a measure to the check the proposed method. The Adjoint method, on the other hand, makes use of the gradient function in Equation (30) to update initial guess value of the parameter $q$ until convergence is reached. Convergence in both cases depends on the minimum norm solution by the method of least squares. A graph showing the Modelled and the observed parameter values at a specific point source, $x_{2}=\frac{1}{2}$ for both the Adjoint method and the PTM are shown in Figures 2 and 3 respectively. Although, there are disparities in the solution between the modelled parameter values and their corresponding observed parameters, the modelled parameter values simulated using the observed data are not far from the true parameter values. From Figure 3, it can be observed from the solution curves that the results obtained using the PTM is better skewed compared to that obtained using the Adjoint method.

The residual norm-error for the two solutions shows that the PTM is more stable with a minimal norm-error value of 2.2350, compared with that of the Adjoint with a norm-error value of 2.6650. The observed and the modelled parameter values using the PTM is shown in Table 1. From Table 1, the variations in the solution of the parameter values are clearly shown. Though, some parameter values approximate to the observed values, other values do not approximate in any way. The difference in the solution estimates between the observed and the modelled parameters for the PTM can be rectified by incorporating some form of regularisation in the solution estimate to stabilise the computations of the solution, and to reduce the error within the solution to the barest minimum.

The mesh flow direction for the PTM is also shown in Figure 4. The essence of the mesh flow direction is to help assess if there are distortions or spikes in the mesh flow plot.
The simulated mesh plot for the PTM shows a smooth flow direction without any spikes or distortions, an indication that the solution can be accepted without any doubt. The colours in the mesh flow plot represent different flow levels at different height. The absolute percentage errors between the observed and the modelled at each parameter point were also determined to ascertain the deviation between the observed and the modeled.

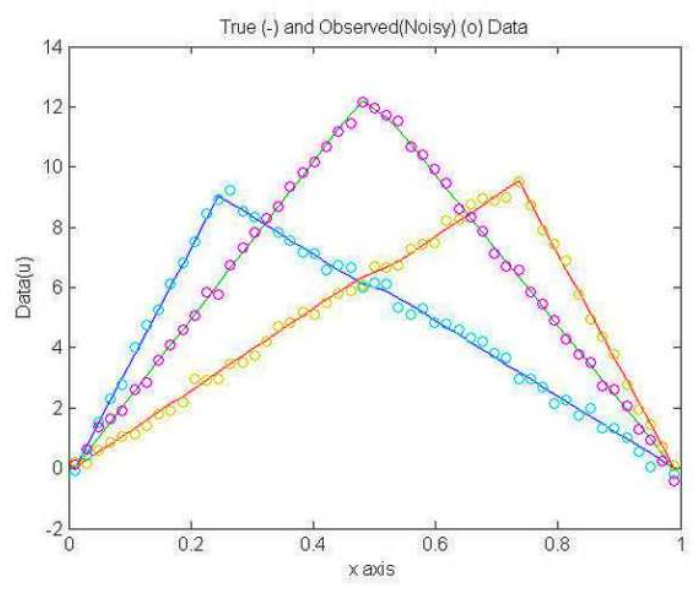

Figure 1. Modelled True and Observed Data.

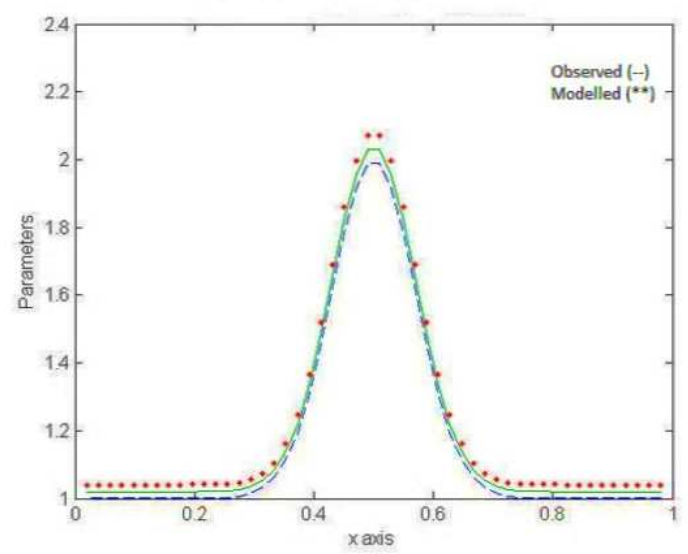

Figure 2. Model Fitness Plots for the Observed and Modelled Parameters Using the Adjoint.

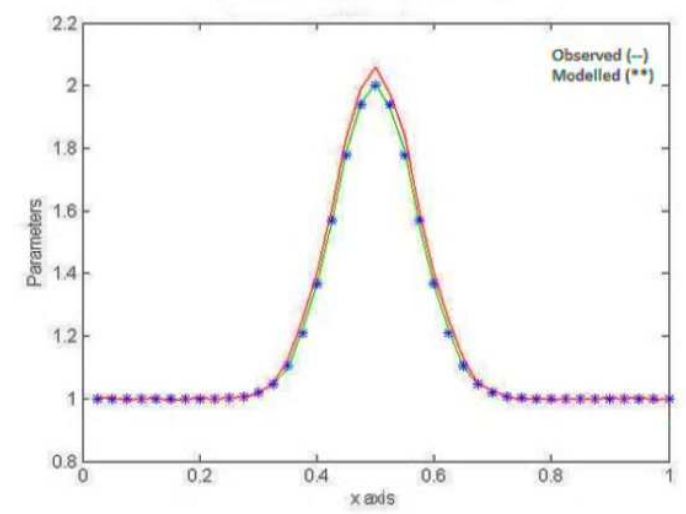

Figure 3. Model Fitness Plots for the Observed and Modelled Parameters Using PTM. 
Table 1. True and Estimated Parameter Values for PTM.

\begin{tabular}{llllllll}
\hline $\begin{array}{l}\text { Iteration } \\
\text { number }\end{array}$ & $\begin{array}{l}\text { Observed } \\
\text { Parameters }\left(\boldsymbol{q}_{\text {obs }}\right)\end{array}$ & $\begin{array}{l}\text { Modelled } \\
\text { Parameters }\left(\boldsymbol{q}_{\boldsymbol{m}}\right)\end{array}$ & Errors (\%) & $\begin{array}{l}\text { Iteration } \\
\text { number }\end{array}$ & $\begin{array}{l}\text { Observed } \\
\text { Parameters }\left(\boldsymbol{q}_{\text {obs }}\right)\end{array}$ & $\begin{array}{l}\text { Modelled } \\
\text { Parameters }\left(\boldsymbol{q}_{\boldsymbol{m}}\right)\end{array}$ & \multicolumn{1}{c}{ Errors $(\%)$} \\
\hline 1 & 1.0000 & 0.9943 & 0.57 & 11 & 1.7788 & 1.8290 & 5.02 \\
2 & 1.0000 & 0.9973 & 0.27 & 12 & 1.3679 & 1.4046 & 3.67 \\
3 & 1.0000 & 1.0001 & 0.01 & 13 & 1.1054 & 1.1289 & 2.35 \\
4 & 1.0001 & 0.9985 & 0.16 & 14 & 1.0183 & 1.0245 & 0.62 \\
5 & 1.0019 & 1.0027 & 0.08 & 15 & 1.0019 & 1.0103 & 0.84 \\
6 & 1.0183 & 1.0153 & 0.30 & 16 & 1.0001 & 0.9949 & 0.91 \\
7 & 1.1054 & 1.1331 & 2.77 & 17 & 1.0000 & 0.9910 & 0.90 \\
8 & 1.3679 & 1.4130 & 4.50 & 18 & 1.0000 & 1.0017 & 0.17 \\
9 & 1.7788 & 1.8304 & 5.16 & 19 & 1.0000 & 1.0022 & 0.22 \\
10 & 2.0000 & 2.0688 & 6.88 & 20 & 1.0000 & 1.0032 & 0.32 \\
\hline
\end{tabular}

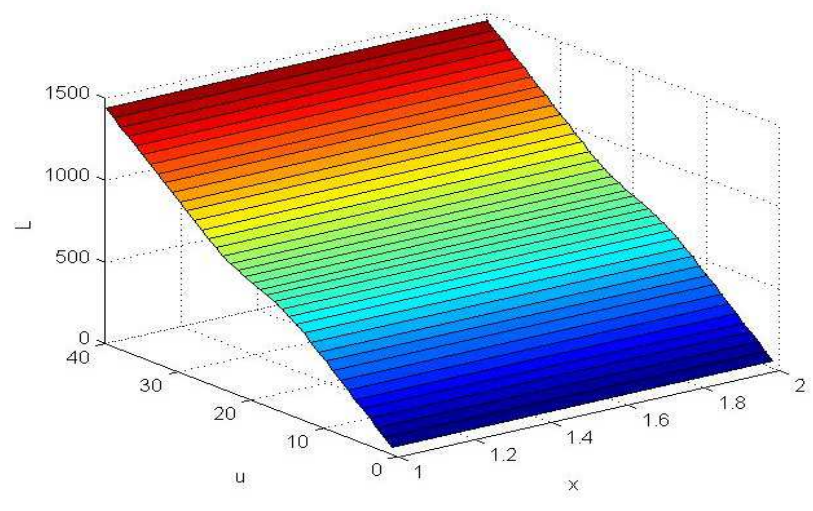

Figure 4. Mesh Flow Directions for PTM.

\section{Numerical Test 2}

Consider a horizontal two-dimensional confined aquifer $\mathrm{ABCD}$, with boundaries $\mathrm{AB}, \mathrm{BC}, \mathrm{CD}$, and $\mathrm{AD}$. If $\mathrm{AD}$ has a constant head boundary $\left(h_{L}=100 \mathrm{~m}\right.$.), and the other heads are impervious, with the length of $\overline{A B}$ being $6500 \mathrm{~m}, \overline{B C}$, $4500 \mathrm{~m}$, and the aquifer being heterogeneous. Suppose also that the aquifer is divided into three zones (AEGD, EFHG, FBCH) with transmissivities $T_{1}, T_{2}, T_{3}$, respectively but their storage coefficients are the same, S. Assume also that there is a pumping well located at the third zone with constant pumping rate $2000 \mathrm{~m}^{3} /$ day, and at the beginning of the pumping, the head is constant everywhere in the aquifer with initial head data value $h_{o}=100 \mathrm{~m}$.

The Numerical Test 2 enumerated above is governed by a non-steady state two-dimensional groundwater flow Equation in Equation (1). The example was adapted from [16], who used the conjugate gradient method to estimate the required parameters. Our aim is to use the proposed PTM coupled with least-squares to recover the same parameters $T_{1}, T_{2}, T_{3}$ and $\mathrm{S}$, and compare the results with the conjugate gradient method values obtained by [16]. In solving the problem, two scenarios were considered:

Scenario 1: Let us assume that the transmissivities and the storativity of the problem are $T_{1}=500 \mathrm{~m}^{2} /$ day, $T_{2}=$ $1000 \mathrm{~m}^{2} /$ day, and $T_{3}=2000 \mathrm{~m}^{2} /$ day and $\mathrm{S}=0.0001$, then the head distribution for the forward problem in Equation (1) can be estimated at specified time steps $\left(t_{1}=0.5, t_{2}=\right.$ 1.0 , and $t_{3}=1.5$ days $)$.
Scenario 2: Using the estimated head data as an observed data, the inverse problem to the forward problem in Scenario 1 is solved to recover the parameters $T_{1}, T_{2}, T_{3}$ and $\mathrm{S}$.

In setting up the grid structure for the problem, the dimensions given are followed. Figure 5 shows the grid structure, the indicated nodal points, as well as the grid size. Each nodal point on the grid corresponds to a flowing well. Generating equations for each zone using the Cell Centred Finite Difference Scheme on the grid structure results in a set of systems of equations. The system of equations generated for the zones AEGD, EFHG, FBCH using Figure 5 are given by the Equations (53), (54) and (55) respectively. The nodal point $u_{12}$ is not considered, since it is assumed to be a pumping well.

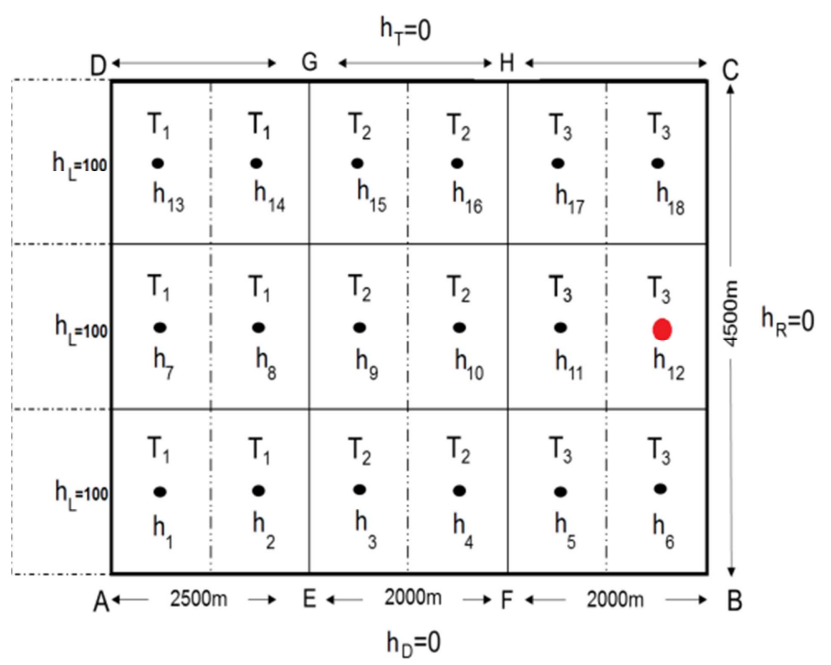

Figure 5. Grid Distributions for Model Problem.

The system of equations in Equations (53)-(55) can be expressed in an algebraic form as depicted by Equation (51). The distributed parameter system in Equation (46) has the coefficient vector $h$ as the head distribution, the parameter $q$ as the unknown parameter of interest with entries $\left[T_{1}, T_{2}, T_{3}, S\right]$, and the function $f$ as a combination of the source term, the pumping rate and the boundary conditions. Given the assumed Transmissivities and the Storage coefficient, the forward problem in Equation (46) can be solved for the head distribution $h$, as shown in Table 2 . 


$$
\begin{aligned}
& \int T_{1}\left(h_{L}-h_{1}\right)+T_{1}\left(h_{7}-h_{1}\right)+T_{1}\left(h_{2}-h_{1}\right)+T_{1}\left(h_{0}-h_{1}\right)=\frac{\mathrm{S}\left(h_{1}^{1}-h_{1}^{0}\right)}{(d x \cdot d y \cdot k \cdot d t)}+\frac{Q}{(d x \cdot d y)} \\
& T_{1}\left(h_{1}-h_{2}\right)+\frac{1}{2}\left(T_{1}+T_{2}\right)\left(h_{3}-h_{2}\right)+T_{1}\left(h_{3}-h_{2}\right)+T_{1}\left(h_{0}-h_{2}\right)=\frac{\mathrm{S}\left(h_{2}^{2}-h_{2}^{1}\right)}{(d x \cdot d y \cdot k \cdot d t)}+\frac{Q}{(d x \cdot d y)} \\
& \frac{1}{2}\left(T_{1}+T_{2}\right)\left(h_{2}-h_{3}\right)+T_{2}\left(h_{4}-h_{3}\right)+T_{2}\left(h_{9}-h_{3}\right)+T_{2}\left(h_{0}-h_{3}\right)=\frac{\mathrm{S}\left(h_{3}^{3}-h_{3}^{2}\right)}{(d x \cdot d y \cdot k \cdot d t)}+\frac{Q}{(d x \cdot d y)} \\
& T_{2}\left(h_{3}-h_{4}\right)+\frac{1}{2}\left(T_{2}+T_{3}\right)\left(h_{5}-h_{4}\right)+T_{2}\left(h_{10}-h_{4}\right)+T_{2}\left(h_{0}-h_{4}\right)=\frac{\mathrm{S}\left(h_{4}^{4}-h_{4}^{3}\right)}{(d x \cdot d y \cdot k \cdot d t)}+\frac{Q}{(d x \cdot d y)} \\
& \frac{1}{2}\left(T_{2}+T_{3}\right)\left(h_{4}-h_{5}\right)+T_{3}\left(h_{6}-h_{5}\right)+T_{3}\left(h_{11}-h_{5}\right)+T_{3}\left(h_{0}-h_{5}\right)=\frac{\mathrm{S}\left(h_{5}^{5}-h_{5}^{4}\right)}{(d x \cdot d y \cdot k \cdot d t)}+\frac{Q}{(d x \cdot d y)} \\
& T_{3}\left(h_{5}-h_{6}\right)+T_{3}\left(h_{6}-h_{6}\right)+T_{3}\left(h_{12}-h_{6}\right)+T_{3}\left(h_{6}-h_{6}\right)=\frac{\mathrm{S}\left(h_{6}^{6}-h_{6}^{5}\right)}{(d x \cdot d y \cdot k \cdot d t)}+\frac{Q}{(d x \cdot d y)} \\
& T_{1}\left(h_{L}-h_{7}\right)+T_{1}\left(h_{1}-h_{7}\right)+T_{1}\left(h_{8}-h_{7}\right)+T_{1}\left(h_{13}-h_{7}\right)=\frac{\mathrm{S}\left(h_{7}^{7}-h_{7}^{6}\right)}{(d x \cdot d y \cdot k \cdot d t)}+\frac{Q}{(d x \cdot d y)} \\
& T_{1}\left(h_{7}-h_{8}\right)+T_{1}\left(h_{2}-h_{8}\right)+\frac{1}{2}\left(T_{1}+T_{2}\right)\left(h_{9}-h_{8}\right)+T_{1}\left(h_{14}-h_{8}\right)=\frac{\mathrm{S}\left(h_{8}^{8}-h_{8}^{7}\right)}{(d x \cdot d y \cdot k \cdot d t)}+\frac{Q}{(d x \cdot d y)} \\
& \left\{\frac{1}{2}\left(T_{1}+T_{2}\right)\left(h_{8}-h_{9}\right)+T_{2}\left(h_{3}-h_{9}\right)+T_{2}\left(h_{10}-h_{9}\right)+T_{2}\left(h_{15}-h_{9}\right)=\frac{\mathrm{S}\left(h_{9}^{9}-h_{9}^{8}\right)}{(d x \cdot d y \cdot k \cdot d t)}+\frac{Q}{(d x \cdot d y)}\right. \\
& T_{2}\left(h_{9}-h_{10}\right)+\frac{1}{2}\left(T_{2}+T_{3}\right)\left(h_{11}-h_{10}\right)+T_{2}\left(h_{4}-h_{10}\right)+T_{2}\left(h_{16}-h_{10}\right)=\frac{\mathrm{S}\left(h_{10}^{10}-h_{10}^{9}\right)}{(d x \cdot d y \cdot k \cdot d t)}+\frac{Q}{(d x \cdot d y)} \\
& \frac{1}{2}\left(T_{2}+T_{3}\right)\left(h_{10}-h_{11}\right)+T_{3}\left(h_{12}-h_{11}\right)+T_{3}\left(h_{5}-h_{11}\right)+T_{3}\left(h_{17}-h_{11}\right)=\frac{\mathrm{S}\left(h_{11}^{11}-h_{11}^{10}\right)}{(d x \cdot d y \cdot k \cdot d t)}+\frac{Q}{(d x \cdot d y)} \\
& \left\{\begin{array}{c}
T_{1}\left(h_{L}-h_{13}\right)+T_{1}\left(h_{14}-h_{13}\right)+T_{1}\left(h_{7}-h_{13}\right)+T_{1}\left(h_{0}-h_{13}\right)=\frac{\mathrm{S}\left(h_{13}^{13}-h_{13}^{12}\right)}{(d x \cdot d y \cdot k \cdot d t)}+\frac{Q}{(d x \cdot d y)} \\
T_{1}\left(h_{13}-h_{14}\right)+\frac{1}{2}\left(T_{1}+T_{2}\right)\left(h_{15}-h_{14}\right)+T_{1}\left(h_{8}-h_{14}\right)+T_{1}\left(h_{0}-h_{14}\right)=\frac{\mathrm{S}\left(h_{14}^{14}-h_{14}^{13}\right)}{(d x \cdot d y \cdot k \cdot d t)}+\frac{Q}{(d x \cdot d y)} \\
\frac{1}{2}\left(T_{1}+T_{2}\right)\left(h_{14}-h_{15}\right)+T_{2}\left(h_{16}-h_{15}\right)+T_{2}\left(h_{9}-h_{15}\right)+T_{2}\left(h_{0}-h_{15}\right)=\frac{\mathrm{S}\left(h_{15}^{15}-h_{15}^{14}\right)}{(d x \cdot d y \cdot k \cdot d t)}+\frac{Q}{(d x \cdot d y)} \\
T_{2}\left(h_{15}-h_{16}\right)+\frac{1}{2}\left(T_{2}+T_{3}\right)\left(h_{17}-h_{16}\right)+T_{2}\left(h_{10}-h_{16}\right)+T_{2}\left(h_{0}-h_{16}\right)=\frac{\mathrm{S}\left(h_{16}^{16}-h_{16}^{15}\right)}{(d x \cdot d y \cdot k \cdot d t)}+\frac{Q}{(d x \cdot d y)} \\
\frac{1}{2}\left(T_{2}+T_{3}\right)\left(h_{16}-h_{17}\right)+T_{3}\left(h_{18}-h_{17}\right)+T_{3}\left(h_{11}-h_{17}\right)+T_{3}\left(h_{0}-h_{17}\right)=\frac{\mathrm{S}\left(h_{17}^{17}-h_{17}^{16}\right)}{(d x \cdot d y \cdot k \cdot d t)}+\frac{Q}{(d x \cdot d y)} \\
T_{3}\left(h_{17}-h_{18}\right)+T_{3}\left(h_{0}-h_{18}\right)+T_{3}\left(h_{12}-h_{18}\right)+T_{3}\left(h_{0}-h_{18}\right)=\frac{\mathrm{S}\left(h_{18}^{18}-h_{18}^{17}\right)}{(d x \cdot d y \cdot k \cdot d t)}+\frac{Q}{(d x \cdot d y)}
\end{array}\right.
\end{aligned}
$$

Table 2. Head Distribution for Forward Problem.

\begin{tabular}{llll}
\hline Node No. & Head (h) at $\mathbf{0 . 5}$ (day) & Node No. & Head (h) at 0.5 (day) \\
\hline 1 & 98.7758453478351 & 10 & 78.0454448836613 \\
2 & 96.6044575770095 & 11 & 57.1107486346670 \\
3 & 93.3890144830225 & 12 & - \\
4 & 86.2008016999414 & 13 & 99.3591675318876 \\
5 & 77.6460997420352 & 14 & 98.9377463132195 \\
6 & 69.4115247730088 & 15 & 94.2775698834049 \\
7 & 98.4989259543307 & 16 & 87.7829178992181 \\
8 & 95.8606930776003 & 17 & 77.9851246418802 \\
9 & 91.5546607586357 & 18 & 69.4962809979700 \\
\hline
\end{tabular}

Using the estimated head distribution $h$ in Table 2, the unknown parameter $q=\left[T_{1}, T_{2}, T_{3}, S\right]$ was recovered by solving the transformed equation in Equation (37) to obtain Equation (56). The generated system obtained in Equation (56), is an over-determined system with a cluster of small singular values within the coefficient matrix. The solution to the over-determined system in Equation (56), sought for either by exact inverse or matrix inversion was found not to be unique. Minimising the over-determined system generated by the PTM results in a unique solution estimate indicated in Table 3. Table 3 gives the results of the identified parameters using the PTM, the Conjugate Gradient method used by [16], 
as well as the observed parameter values.

$\left[\begin{array}{cccc}0.0000021400 & 0 & 0 & 0.0000013057 \\ 3.2154441474 & -1.6077215469 & 0 & 5.4328678767 \\ 1.6077215469 & -0.8038594434 & 0 & 0.0000088146 \\ 0 & 8.5547032879 & -4.2773509789 & 0.0000183989 \\ 0 & 4.2773509789 & -2.1386748394 & 0.0000298052 \\ 0 & 0 & 0.0000006499 & 0.0000407846 \\ 0.0000021399 & 0 & 0 & 0.0000016011 \\ 4.3060344522 & -2.1530161594 & 0 & 0.0000044152 \\ 2.1530161594 & -1.0765067497 & 0 & 0.0000112604 \\ 0 & 20.9346975823 & -10.4673481244 & 0.0000292727 \\ 0 & 10.4673481244 & -5.2336733955 & 0.0000571856 \\ 0.0000021400 & 0 & 0 & 0.0000006835 \\ -1.0622514867 & 0.5311268433 & 0 & 0.0000011330 \\ 2.3300882149 & -1.1650427774 & 0 & 0.0000076299 \\ 0 & 9.7977945573 & -4.8988966286 & 0.0000162894 \\ 0 & 4.8988966286 & -2.4494476643 & 0.0000293531 \\ 0 & 0 & 0.0000006500 & 0.0000406716\end{array}\right]\left[\begin{array}{c}2 / 1875 \\ 2 / 1875 \\ 1 / 750 \\ 1 / 750 \\ 1 / 750 \\ T_{2} \\ 1 / 750 \\ 2 / 1875 \\ 2 / 1875 \\ 1 / 750 \\ 1 / 750 \\ 1 / 750 \\ 2 / 1875 \\ 2 / 1875 \\ 1 / 750 \\ 1 / 750 \\ 1 / 750 \\ 1 / 750\end{array}\right]$

Table 3. Observed and Identified Parameters.

\begin{tabular}{llll}
\hline Parameters & $\begin{array}{l}\text { Observed } \\
\text { Values }\end{array}$ & PTM & $\begin{array}{l}\text { Conjugate Gradient } \\
\text { Method (CG) }\end{array}$ \\
\hline$T_{1}$ & 500 & 503.891 & 525 \\
$T_{2}$ & 1000 & 1007.783 & 972 \\
$T_{3}$ & 2000 & 2011.567 & 2078 \\
$\mathrm{~S}$ & $1 \times 10^{-4}$ & $9.925 \times 10^{-5}$ & $1.006 \times 10^{-4}$ \\
\hline
\end{tabular}

Although, none of the estimated solutions approximate the observed parameter values, the values obtained by the PTM is more stable with a relative norm-error value of

14.47566340, compared with that of the Conjugate Gradient method with a relative norm-error value of 86.56211640 . When the time step is increased from $t=0.5$ days to $t=1.0$ and 1.5 days respectively and solved using the generated system of Equations (53)-(55), the head data at these time steps are obtained. Using the head data at these time steps, Equation (56) is solved again by going through same procedure for the parameter $q$. The results obtained are indicated in Table 4. The result shows much consistency in values after a time step of $t=1.0$ day.

Table 4. True and Identified Parameters at Different Time Steps.

\begin{tabular}{lllllll}
\hline Parameters & Observed Values & PTM at $\mathbf{t}=\mathbf{0 . 5}$ & PTM at t=1.0 & PTM at $\mathbf{t}=\mathbf{1 . 5}$ & Costate Method & Conjugate Gradient Method (CG) \\
\hline$T_{1}$ & 500 & 503.944 & 497.603 & 497.603 & 507.663 & 525 \\
$T_{2}$ & 1000 & 1007.589 & 995.207 & 995.207 & 1011.253 & 972 \\
$T_{3}$ & 2000 & 2011.777 & 1990.413 & 1990.413 & 2020.111 & 2078 \\
$\mathrm{~S}$ & $1 \times 10^{-4}$ & $9.925 \times 10^{-4}$ & $1.935 \times 10^{-4}$ & $2.902 \times 10^{-5}$ & $1.9 \times 10^{-4}$ & $10^{-5}$ \\
\hline
\end{tabular}

\section{Discussions}

The proposed PTM for recovering inverse flow parameters has been presented. The Partial Differential Equation (PDE) describing the inverse flow model of interest was first discretised by either the Finite Difference Method (FDM) and the Cell Centred Finite Difference (CCFD) method. The discretisation led to a Parameter-dependent system or a distributed parameter system. The PTM was sought to transform the distributed parameter system to a system that depended on the state vector. The method proposed was tested numerically on one and two-dimensional flow models governed by PDEs. The results revealed that the PTM can be used to recover inverse flow parameters. To assess the accuracy and the stability of the proposed method, the Adjoint method, and the Conjugate Gradient Method were adapted as a measure to the PTM. When the adapted methods and the PTM were simulated numerically on a 1D and 2D test problems, the results revealed that the PTM is more stable with a residual norm-error value of 2.23500 for the $1 \mathrm{D}$ test problem compared with that of the Adjoint method with a norm-error value of 2.66500. For the 2D test case, the results also revealed that the PTM was stable with a residual norm-error value of 10.98310 compared with that of the Conjugate Gradient method with value of 86.56212 . Although, the estimated parameter values obtained by the PTM approximate the true parameter values, the disparities in solution between the true and the estimated parameter values for both numerical tests considered were very high. Also, for the proposed PTM, the time component at times needs to be varied to obtain realistic solution estimates. In the studied 2D problem, for instance, the initial time step did not yield an approximate solution. When the time step was varied from $t=0.5$ to $t=1.0$ and 1.5 days, the results showed better consistency. Though, the estimated parameter values using the PTM approximated to the observed parameter values, the relative norm error value of the solution was observed to be a bit high. For a smooth solution estimate with minimal norm-error, some form of regularisation needs to be incorporated into the solution to stabilise the system. 


\section{Conclusion}

Although Optimisation techniques have been explored, the study revealed that the PTM is capable of yielding realistic solution estimates compared with the studied optimisation methods. Thus, when a mathematical model is available, and measurements data are available, a solution estimate is possible. A smooth solution estimate requires some form of regularisation. Further investigation should be conducted by incorporating a regularisation term or a penalty function to stabilise the computations of the flow parameters to improve the convergence.

\section{References}

[1] Beven, K. J. (2006), A manifesto for the equifinality thesis, J. Hydrol., 320 (1-2), 18-36.

[2] Bruckner G, Handrock-Meyer S, and Langmach, H (1998), An inverse problem from 2D ground-water modelling, Inverse Problems, 14 (4): 835-851.

[3] Cacuci, D. G., (1981), Sensitivity Theory for Nonlinear Systems: Nonlinear Functional Analysis Approach, J. Math. Phys., 22, 2794.

[4] Carter RD, Kemp LF, Pierce AC, and Williams DL (1974) Performance Matching with constraints, Society of Petroleum Engineering Journal, 14 (02), 187-196.

[5] Chavent G, Dupuy M, and Lemmonier P (1975) History Matching by Use of Optimal Theory, Society of Petroleum Engineers Journal, 15 (01), 74-86.

[6] Dietrich C. R, and Newsam G. N (1990), Sufficient Conditions for Identifying Transmissivity in a Confined Aquifer, Inverse Problems, 6 (3): L21-L28.

[7] Irsa, J, and Zhang Y (2012), A Direct Method of Parameter Estimation for Steady State Flow in Heterogeneous Aquifers with Unknown Boundary Conditions, Water Resources Research, 48: W09526.

[8] Jacquard, P., and C. Jain, (1965), Permeability Distribution from field Pressure Data, Trans Society of Petroleum Engineers, Vol. (54), 281-294.

[9] Knowles I, and Yan A (2007) The reconstruction of groundwater parameters from head data in an unconfined aquifer, Journal of Computational and Applied Mathematics, 208 (1): $72-81$.

[10] Knowles I, Le T, and Yan A (2004) On the recovery of multiple flow parameters from transient head data, Journal of Computational and Applied Mathematics, 169 (1): 1-15.

[11] LeVeque RJ. (2007) Finite difference methods for ordinary and partial differential equations, Steady state and time dependent problems, Society for Industrial and Applied Mathematics, Philadelphia, USA, 341p.

[12] Neuman, S. P, Fogg, G. E and Jacobson E. A (1980), A statistical approach to the inverse problem of aquifer hydrology, Case study, Water Resources Research, Vol. 16, No. 1, pp. 33-58.
[13] Neuman, SP. and S. Yakowitz. (1979), "A statistical approach to the inverse problem of aquifer hydrology 1Theory", Water Resour. Res., Vol. 15, No. 4, pp. 845-860.

[14] Nguyen VT, Nguyen HT, Tran TB, Vo AK (2015) On an inverse problem in the parabolic equation arising from groundwater pollution problem, Boundary Value Problems, 67, DOI 10.1186/s13661-015-0319-3.

[15] Oblow EM (1978) Sensitivity Theory for Reactor ThermalHydraulics Problems, Nuclear Science and Engineering, 68 (3), 322-337.

[16] Sun NZ (1999), Inverse Problems in Groundwater Modeling, 1st edn., Kluwer Academic Publishers, Boston, USA, 337p.

[17] Sun, N. Z., and Yeh, WWG (1990a), Coupled inverse problems in groundwater modeling: 1 . Sensitivity analysis and parameter identification, Water Resour. Res., 26 (10), $2507-$ 2525 .

[18] Sun, NZ. and Yeh, WG. (1990), "Coupled Inverse Problems in Groundwater Modelling: Sensitivity Analysis and Parameter Identification", Water Resources Research, Vol. 26, No. 10, pp. 2507-2525.

[19] Sun, NZ., and Yeh, WWG (1990b), Coupled inverse problems in groundwater modeling: 2. Identifiability and experimental design, Water Resour. Res., 26 (10), 2527-2540.

[20] Sykes, JF., Wilson, JL and Andrews, RW (1985), Sensitivity analysis for steady state groundwater flow using adjoint operators, Water Resour. Res., 21 (3), 359-371.

[21] Tarantola, A., (1987), Inverse Problems Theory: Methods for Data Fitting and Model Parameters Estimation, Elsevier, New York.

[22] Townley, LR and Wilson, JL (1985), Computationally Efficient Algorithms for Parameter Estimation and Uncertainty Propagation in numerical Models of groundwater flow. Water Resources Research, 21 (12), 1851-1860.

[23] Vainikko G, and Kunisch K (1993) Identifiability of the transmissivity coefficient in an elliptic boundary value problem, Zeitschrift für Analysis und ihre Anwendungen, 12 (2): 327-341.

[24] Vemuri, V., and W. J. Karplus (1969), Identification of nonlinear parameters of ground water basins by hybrid computation, Water Resour. Res., 5 (1), 172-185.

[25] Vogel CR (1999) Sparse matrix computations arising in distributed parameter identification, SIAM J. Matrix Anal. Appl. 20 (4): 1027-1037.

[26] Vogel CR (2002) Computational Methods for Inverse Problems (Frontiers in Applied Mathematics), 1st edn., Society for Industrial and Applied Mathematics, Philadelphia, USA, 183p.

[27] Wilson, J. L., and D. E. Metcalfe (1985), Illustration and verification of adjoint sensitivity for steady state groundwater flow, Water Resour. Res., 21 (11), 1602-1610.

[28] Yeh WWG (2015) Review: Optimisation methods for groundwater modelling and management, Hydrogeology Journal, 23 (6): 1051-1065. 